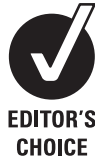

- Additional supplemental fig 1 is published online only at http:// adc.bmj.com/content/vol94/ issue8

${ }^{1}$ Department of General Paediatrics, Princess Margaret Hospital for Children, Perth, Western Australia; ${ }^{2}$ Department of Respiratory Medicine, Princess Margaret Hospital for Children, Perth, Western Australia; ${ }^{3}$ School of Paediatrics and Child Health, University of Western Australia, Perth, Western Australia; ${ }^{4}$ Ambulatory Care Unit, Princess Margaret Hospital for Children, Perth, Western Australia; ${ }^{5}$ Clinical Research, Princess Margaret Hospital for Children, Perth,

Western Australia

Correspondence to: Dr A Martin, andrew.martin@ health.wa.gov.au

Accepted 26 September 2008 Published Online First

14 October 2008

\title{
Home oxygen for children with acute bronchiolitis
}

\author{
S W Tie, ${ }^{1} \mathrm{G}$ L Hall,,${ }^{2,3}$ S Peter, ${ }^{4}$ J Vine, ${ }^{4}$ M Verheggen, ${ }^{2}$ E M Pascoe, ${ }^{5}$ A C Wilson, ${ }^{2,3}$ \\ G Chaney, ${ }^{1,3}$ S M Stick, ${ }^{2,3}$ A C Martin ${ }^{1,3}$
}

\section{ABSTRACT}

A prospective randomised controlled pilot study was performed comparing home oxygen therapy with traditional inpatient hospitalisation for children with acute bronchiolitis. Children aged 3-24 months with acute bronchiolitis, still requiring oxygen supplementation $24 \mathrm{~h}$ after admission to hospital, were randomly assigned to receive oxygen supplementation at home with support from "hospital in the home" (HiTH) or to continue oxygen supplementation in hospital. 44 children (26 male, mean age 9.2 months) were recruited (HiTH $n=22$ ) between 1 August and 30 November 2007. Only one child from each group was readmitted to hospital and there were no serious complications. Children in the HiTH group spent almost 2 days less in a hospital bed than those managed as traditional inpatients: HiTH $55.2 \mathrm{~h}$ (interquartile range (IOR) 40.3-88.9) versus in hospital $96.9 \mathrm{~h}$ (IOR 71.2-

147.2) $p=0.001$. Home oxygen therapy appears to be a feasible alternative to traditional hospital oxygen therapy in selected children with acute bronchiolitis.

Acute bronchiolitis is a common reason for hospitalisation in infants in developed countries, ${ }^{1}$ with treatment being essentially supportive. Although the need for supplemental oxygen is generally considered to be an absolute indication for hospitalisation, ${ }^{2}$ Bajaj et $a l^{3}$ recently demonstrated that children with acute bronchiolitis can be managed safely with home oxygen therapy. The broad application of this approach, however, has not been tested.

The development of nurse-led, home-based care has allowed children with a variety of illnesses to be managed safely at home rather than in hospital. ${ }^{45}$ Whereas managing children with acute illnesses at home is not a new strategy, it is an increasingly attractive alternative to traditional inpatient hospitalisation. Home oxygen therapy is a well-accepted option for children with chronic respiratory problems, ${ }^{6}$ but reports of home oxygen therapy for children with acute respiratory problems are limited. We sought to determine the feasibility and safety of home oxygen therapy for children with acute bronchiolitis compared with traditional inpatient hospitalisation.

\section{METHODS}

\section{Setting and subjects}

We conducted a prospective, randomised controlled pilot study comparing home oxygen therapy for children with acute bronchiolitis requiring oxygen supplementation with traditional inpatient hospitalisation. All children aged 3-24 months, admitted to Princess Margaret Hospital for Children (PMH), Perth, Western Australia, with a clinical diagnosis of acute bronchiolitis, who required oxygen therapy and satisfied all inclusion and exclusion criteria (table 1) were eligible. Children were recruited over a single Australian bronchiolitis season, between 1 August 2007 and 30 November 2007.

\section{Study protocol}

Families of eligible children (table 1) were approached and following informed consent children were randomly assigned to continue traditional inpatient care (hospital group) or to continue oxygen therapy at home (hospital in the home (HiTH) group). Researchers were blinded and only following informed consent was the management allocation revealed.

Following randomisation children underwent a modified "safety in air test", designed to provide some degree of reassurance that if oxygen supplementation were interrupted for an extended period the child would not develop sudden, life-threatening hypoxia. ${ }^{6}$ The test involved continuous monitoring of pulse oxygen saturation $\left(\mathrm{SpO}_{2}\right)$ levels and clinical status of the child, while in room air, over a period of 20 minutes. If $\mathrm{SpO}_{2}$ remained at $80 \%$ or greater the child was considered to pass the test.

Children randomly assigned to the hospital group had their weaning of supplemental oxygen and the time of discharge from hospital managed by their paediatrician, independently of the study investigators. Standard management of children with acute bronchiolitis at our institution involves supportive care only, with supplemental oxygen therapy provided if $\mathrm{SpO}_{2}$ levels are less than $93 \%$.

Parents of children randomly assigned to the HiTH group were assessed for suitability and safety of providing care at home, were educated on home oxygen use and instructed on how to observe their children for signs of clinical deterioration. Children were reviewed by a HiTH nurse within $12 \mathrm{~h}$ of hospital discharge and received a minimum of two visits, in addition to one phone contact with the parents in every $24-\mathrm{h}$ period. At each visit, if $\mathrm{SpO}_{2}$ was greater than $92 \%$, oxygen was reduced as follows: 1 to $0.75 \mathrm{l} /$ minute, 0.75 to $0.5 \mathrm{l} /$ minute, 0.5 to $0.25 \mathrm{l} /$ minute, 0.25 to $0.125 \mathrm{l} /$ minute and 0.125 to $0.06 \mathrm{l} /$ minute and $\mathrm{SpO}_{2}$ monitored for a further 15 minutes. If $\mathrm{SpO}_{2}$ remained greater than $92 \%$ the child remained on this oxygen flow until the next visit. Once the child reached $0.06 \mathrm{l} /$ minute a 15 -minute trial in air was conducted and if $\mathrm{SpO}_{2}$ remained greater than 92\% the child was discharged.

Criteria for readmission to hospital were: (1) Oxygen requirement increased to more than $1 \mathrm{l} /$ minute to maintain $\mathrm{SpO}_{2}$ at greater than $92 \%$; (2) apnoeic episode; (3) feeding less than $50 \%$ of 
Table 1 Inclusion and exclusion criteria

\begin{tabular}{|c|c|}
\hline Inclusion criteria & Exclusion criteria \\
\hline 3-24 Months of age (corrected gestation) & $\begin{array}{l}\text { Pre-existing cardiac, pulmonary (including chronic } \\
\text { lung disease of infancy, cystic fibrosis and } \\
\text { congenital or acquired airway anomalies) and } \\
\text { neuromuscular disorders }\end{array}$ \\
\hline Clinical diagnosis of acute bronchiolitis & History of apnoea \\
\hline Adequate feeding ( $\geqslant 50 \%$ normal) and hydration & Prematurity $<34$ weeks' gestation \\
\hline \multicolumn{2}{|l|}{ Oxygen saturation $>92 \%$ on $\leqslant 1 \mathrm{l} /$ minute nasal cannula oxygen. } \\
\hline \multicolumn{2}{|l|}{ Observed and clinically stable for $\geqslant 24 \mathrm{~h}$ in hospital } \\
\hline \multicolumn{2}{|l|}{ Pass modified "safety in air test" } \\
\hline \multicolumn{2}{|l|}{$\begin{array}{l}\text { Caregivers must be counselled about risk of smoking around a child receiving } \\
\text { oxygen supplementation }\end{array}$} \\
\hline \multicolumn{2}{|l|}{ Caregivers must be adequately educated about home oxygen } \\
\hline \multicolumn{2}{|l|}{$\begin{array}{l}\text { HiTH nurses able to visit at home at least twice daily, in addition to daily phone } \\
\text { call }\end{array}$} \\
\hline Paediatrician agrees that child is eligible for recruitment in study & \\
\hline
\end{tabular}

normal with clinical evidence of dehydration; (4) parents or treating paediatrician requested withdrawal of the child from the study.

The primary outcome measure was readmission to hospital within 7 days of discharge home and the secondary outcome measure was total days spent in a hospital bed. The study was approved by the Princess Margaret Hospital Ethics Committee.

\section{Statistical analysis}

A successful discharge was defined as not requiring readmission to hospital within 7 days and a 10\% difference in readmission rates between groups was considered to be clinically significant. Using a minimum success rate of $100 \%$ for the hospital group and $90 \%$ for $\mathrm{HiTH}$, we estimated that 180 children (90 in each group) would be needed to demonstrate a difference, with $80 \%$ power and 5\% significance. Continuous data are expressed as median (interquartile range; IOR) and group differences compared with Wilcoxon rank sum test. Categorical data are shown as number (percentage) and compared with Fisher's exact tests.

\section{RESULTS}

Forty-four of 58 eligible children were enrolled (table 2). Seven families were not approached as the children met the inclusion criteria outside normal working hours, four lived outside the HiTH catchment area and three refused consent (see supplemental fig 1 available online only).

One child from each group (4.5\%, 95\% CI 0.1 to 22.8 ), required readmission to hospital. The child from the $\mathrm{HiTH}$ group was readmitted with dehydration secondary to viral gastroenteritis, with no change in respiratory status and was managed with nasogastric rehydration and oxygen supplementation. The child from the hospital group was readmitted with bacterial pneumonia requiring oxygen supplementation and antibiotics. Both children made full and uneventful recoveries.

Children in the HiTH group spent significantly less time in a hospital bed (55.2 h, IOR 40.3-88.9) than those in the hospital group (96.9 h, IOR 71.2-147.2, $\mathrm{p}=0.001$ ). HiTH children received five (range four to 18 ) home visits from the time of hospital to HiTH discharge, with two (range one to six) phone contacts per patient.

\section{DISCUSSION}

This study suggests that home oxygen therapy is a feasible alternative to traditional hospital care in selected children with uncomplicated acute bronchiolitis.

No differences in readmission rates between the $\mathrm{HiTH}$ and hospital groups were noted, with only one of $22(<5 \%)$ children in each group readmitted. The present study is limited by its small size, a result of funding and operational constraints. Whereas further studies with increased numbers are required, we believe that carefully selected children with acute bronchiolitis requiring oxygen therapy can be safely managed at home by their parents with HiTH support.

We demonstrated a significant reduction in the duration of hospital admission in the HiTH group compared with hospitalbased care. This decreased length of stay may lead to improvements in service efficiency, including increased availability of hospital beds at a time when the demand for resources is at a peak.

In contrast to the study by Bajaj et $a l^{3}$, we admitted all children to hospital for at least $24 \mathrm{~h}$ to ensure that they were feeding adequately and were clinically stable, a policy felt to be more acceptable to paediatricians. Furthermore, in the study by Bajaj et $\mathrm{al}^{3}$, primary care physicians supervised the weaning of oxygen, whereas this study utilised a nurse-led home-based

Table 2 Demographic characteristics of each group

\begin{tabular}{lccc}
\hline & Hospital group & HiTH group & p Value \\
\hline No of children & 22 & 22 & \\
Median age, months (range) & $8.7(3.0-18.0)$ & $8.5(3.0-17.6)$ & 0.83 \\
Males, $\mathrm{n}(\%)$ & $10(45.4 \%)$ & $16(72.7 \%)$ & 0.12 \\
Oxygen saturation on ED arrival (median) & $93 \%(81-99)$ & $94 \%(88-99)$ & 0.70 \\
RSV positive & $16(72.7 \%)$ & $11(50 \%)$ & 0.12 \\
Parental smoking, $\mathrm{n}(\%)$ & $7(31.8 \%)$ & $6(27.3 \%)$ & 0.28 \\
\hline
\end{tabular}

ED, emergency department; HiTH, hospital in the home; RSV, respiratory syncytial virus. 
service, a design more reflective of paediatric ambulatory care services available in Australia and the UK.

The lack of an effective treatment to change the natural history of acute bronchiolitis means innovative approaches are required to decrease the health and economic burden of the commonest cause of hospitalisation for infants in developed countries. Using carefully considered inclusion and exclusion criteria, home oxygen therapy is a feasible option in the management of children with acute bronchiolitis. This practice could potentially be established in any paediatric unit that is supported by a nurse-led HiTH programme.

Acknowledgements: The authors would like to thanks the children and families who participated in the study, as well as the ambulatory care unit and general paediatricians at Princess Margaret Hospital for Children.

Funding: This study was supported by a grant from the Western Australian State Health Research Advisory Council.
Competing interests: None

Ethics approval: The study was approved by the Princess Margaret Hospital Ethics Committee.

Patient consent: Obtained.

\section{REFERENCES}

1. Shay DK, Holman RC, Newman RD, et al. Bronchiolitis-associated hospitalizations among US children, 1980-1996. JAMA 1999;282:1440-6.

2. Scottish Intercollegiate Guidelines Network (SIGN). Bronchiolitis in children: a national clinical guideline. (SIGN publication no 91). NHS Quality Improvement Scotland. November 2006.

3. Bajaj L, Turner CG, Bothner J. A randomized trial of home oxygen therapy from the emergency department for acute bronchiolitis. Pediatrics 2006;117:633-40.

4. Cooper C, Wheeler DM, Woolfenden SR, et al. Specialist home-based nursing services for children with acute and chronic illnesses. Cochrane Database Syst Rev 2006; (4):CD004383.

5. Meates M. Ambulatory paediatrics - making a difference. Arch Dis Child 1997:76:468-73

6. Saletti A, Stick S, Doherty D, et al. Home oxygen therapy after preterm birth in Western Australia. J Paediatr Child Health 2004;40:519-23.

\section{Images in paediatrics}

\section{A plethoric palm}

A 5-year-old boy presented with a red hand since birth. On examination, his right palm was red, plethoric and slightly larger when compared with his left palm (fig 1). A diffuse capillary haemangioma of the right palm extending proximally to involve the entire upper limb and the right pectoral region was the cause for the marked plethora. Mild hypertrophy of the right upper limb and a few tortuous veins over the right pectoral region were noted (fig 2), and hence a diagnosis of Klippel-Trenaunay syndrome was considered. KlippelTrenaunay syndrome is a rare, sporadic, complex malformation characterised by the clinical triad of (1) capillary malformations (port wine stain), (2) soft tissue and bone hypertrophy or, occasionally, hypotrophy of usually one lower limb and (3) atypical, mostly lateral varicosity. ${ }^{1}$ Although lower-limb involvement is very common among the patients with this syndrome, upper-limb involvement has been observed. ${ }^{2}$ The

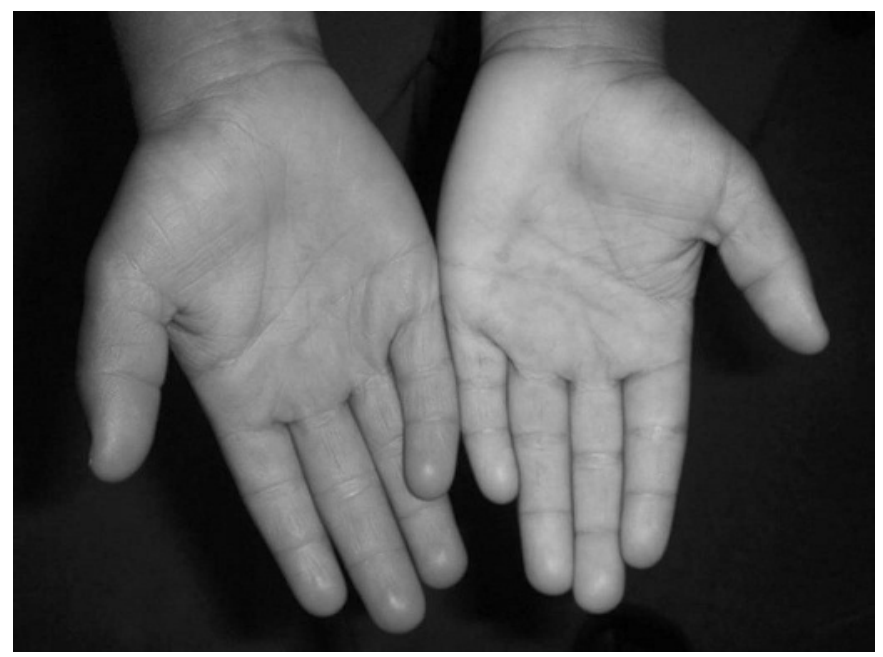

Figure 1 Comparison of the patient's left and right hand.

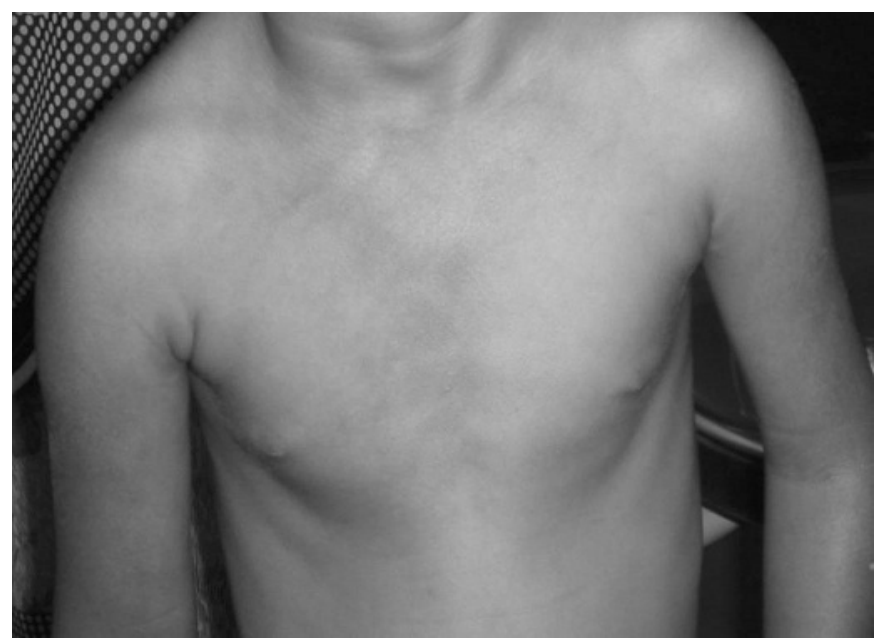

Figure 2 Mild hypertrophy of the right upper limb and a few tortuous veins over the right pectoral region of the patient.

striking colour contrast of the palms and upper-limb involvement make this case interesting.

\section{B Adhisivam}

Correspondence to: Dr B Adhisivam, Jawaharlal Institute of Postgraduate Medical Education and Research, Pondicherry 605 006, India; adhisivam1975@yahoo.co.uk

Competing interests: None.

Ethics approval: Ethics approval was provided by MGMCRI.

Patient consent: Obtained from the parents.

Accepted 25 April 2009

Arch Dis Child 2009;94:643. doi:10.1136/adc.2009.164269

\section{REFERENCES}

1. Gloviczki P, Driscoll DJ. Klippel-Trenaunay syndrome: current management. Phlebology 2007;22:291-8.

2. Akcali C, Inaloz S, Kirtak N, et al. A case of Klippel-Trenaunay syndrome involving only upper limbs. G Ital Dermatol Venereol 2008;143:267-9. 